\title{
The prognostic impact of a combined carbonic anhydrase IX and Ki67 signature in oral squamous cell carcinoma
}

\author{
A C Klimowicz ${ }^{1,2,5}$, P Bose ${ }^{1}$, S K Petrillo ${ }^{2}$, A M Magliocco ${ }^{1,2,6}$, J C Dort ${ }^{\star, 3}$ and N T Brockton ${ }^{\star 1,4}$ \\ ${ }^{1}$ Department of Oncology, University of Calgary, 1331 - 29 Street NW, Calgary, Alberta, Canada T2N 4N2; ${ }^{2}$ Functional Tissue Imaging \\ Unit, Translational Laboratories, Tom Baker Cancer Centre, 1331 - 29 Street NW, Calgary, Alberta, Canada T2N 4N2; ${ }^{3}$ Division of \\ Otolaryngology-Head and Neck Surgery, University of Calgary, 1403 - 29 Street NW, Calgary, Alberta, Canada T2N 2T9 and ${ }^{4}$ Department \\ of Population Health Research, Alberta Health Services - Cancer Care, 1403 - 29 Street NW, Calgary, Alberta, Canada T2N 2T9
}

Background: Tumour hypoxia is associated with impaired apoptosis, resistance to therapy and poor prognosis. We previously reported that high stromal expression of the endogenous marker of hypoxia, carbonic anhydrase IX (CAIX), is associated with significantly reduced survival in oral squamous cell carcinoma (OSCC). In addition to hypoxia, CAIX expression is regulated by proliferation-associated signalling. We hypothesised that incorporating Ki67, a proliferation marker, into our existing CAIX-based stratification of OSCC would identify patients with the least favourable prognosis.

Methods: Surgically resected tumours from 60 OSCC patients were analysed for CAIX, Ki67 and BAX expression using fluorescence immunohistochemistry and automated quantitative analysis (AQUA).

Results: In patients expressing high stromal CAIX (sCAIX), stratification by tumour Ki67 expression revealed significantly distinct survival outcomes $(P=0.005)$. In our OSCC cohort, below-median Ki67 and top-quartile sCAIX expression (Kib7 ${ }^{\text {lo }}$ SCAIX ${ }^{\text {hi }}$ ) were associated with significantly worse disease-specific survival in univariate (HR 7.2 (2.5-20.4), $P=0.001$ ) and multivariate (HR 4.2 $(1.4-12.8), P=0.011)$ analyses. Hypoxia is associated with decreased BAX expression; the Ki67 ${ }^{\mathrm{lo}} \mathrm{SCAIX} \mathrm{X}^{\mathrm{hi}}$ group was more strongly associated with low BAX expression than high sCAIX alone.

Conclusion: These data suggest that combined analysis of tumour Ki67 and sCAIX expression may provide a more clinically relevant assessment of tumour hypoxia in OSCC.

Oral squamous cell carcinoma (OSCC) is the most common head and neck cancer, annually accounting for more than 250000 new cases worldwide (Jemal et al, 2011). Despite advances in diagnostic, surgical and chemoradiation techniques, only $50 \%$ of OSCC patients survive beyond 5 years (Campana and Meyers, 2006); in fact, survival rates in OSCC have remained unchanged for the last five decades. A better understanding of the underlying biological mechanisms, and their impact on OSCC prognosis, could provide more effective treatments and improve survival rates.
Hypoxia is rare in normal oral epithelial tissue (Chen et al, 2012) but is common within the tumour microenvironment. Hypoxic tumours are associated with tumour invasiveness, metastasis and an overall poor prognosis (Janssen et al, 2005; Wilson and Hay, 2011; Toustrup et al, 2012). The effectiveness of traditional chemotherapy and radiotherapy is dependent on inducing apoptosis in tumour cells; however, hypoxia selects for tumour cells with reduced sensitivity to apoptotic stimuli by suppressing pro-apoptotic BAK/BAX signalling (Erler et al, 2004;

*Correspondence: Dr NT Brockton; E-mail: nigel.brockton@albertahealthservices.ca or Dr JC Dort; E-mail: jdort@ucalgary.ca

${ }^{5}$ Present address: Boehringer Ingelheim Pharmaceuticals, Inc., 900 Ridgebury Road, Ridgefield, CT 06877, USA.

${ }^{6}$ Present address: H. Lee Moffitt Cancer Center \& Research Institute, 12902 Magnolia Drive, Tampa, FL 33612, USA.

Received 1 July 2013; revised 26 July 2013; accepted 13 August 2013;

published online 5 September 2013

(c) 2013 Cancer Research UK. All rights reserved 0007-0920/13

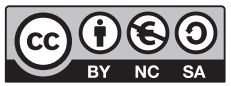

BIC $\cap$ 
Nelson et al, 2004). Hypoxic sensitisers and hypoxia-specific toxins offer significant potential for improving treatment efficacy in OSCC. However, identifying hypoxic tumours in the clinical setting is presently a barrier to the effective application of hypoxiatargeting/sensitising therapeutics (Wilson and Hay, 2011).

Several methods have been used to identify hypoxic tumours; however, there is currently no single gold-standard technique. Direct measurement by oxygen electrode is sensitive but must reconcile substantial intra-tumoural heterogeneity and anatomic restrictions for access. Exogenous markers such as bioreductive drugs (e.g., pimonidazole) are convenient to administer, and easily imaged, but the extent of hypoxia required for activation may not necessarily reflect clinically relevant tumour hypoxia and may be subject to incomplete distribution due to ineffective perfusion of some tumour areas (Wilson and Hay, 2011). Immunohistochemistry (IHC)-based detection of tumour hypoxia by staining for endogenous markers of hypoxia (EMH) is both cost effective and clinically feasible, but there is a lack of agreement as to which EMH most reliably indicate an adverse tumour phenotype and poor prognosis (Sorensen et al, 2005). Expression of the EMH carbonic anhydrase IX (CAIX) has been shown to be consistently associated with prognosis across several cancer sites, including head and neck squamous cell carcinoma (HNSCC; Hoogsteen et al, 2007; Kaluz et al, 2009; Brockton et al, 2011, 2012; McDonald et al, 2012). However, many studies have reported discrepancies between CAIX expression and other measures of tissue hypoxia, such as $\mathrm{pO}_{2}$ (Kaluz et al, 2009), or bioreductive drug markers, such as pimonidazole (Kaanders et al, 2002; Mayer et al, 2005; Wilson and Hay, 2011). This discordant distribution is likely due to differences in the threshold of activation for bioreductive drugs (exogenous) vs endogenous markers, the half-life of other hypoxic indicators (e.g., HIF1 $\alpha$ ), fluctuating concentration of oxygen within tumour tissues (Sobhanifar et al, 2005) and oxygen-independent regulation of CAIX expression. We propose that the prognostic value of CAIX will be improved by considering additional factors that influence CAIX expression.

Carbonic anhydrase IX expression is regulated by the transcription factor HIF $1 \alpha$ (Sowter et al, 2003). HIF1 $\alpha$ stability and activity are principally regulated by the $\mathrm{pO}_{2}$ levels within cells; however, increased HIF1 $\alpha$ stability and transcriptional activity can also be achieved under normoxic/mildly hypoxic conditions by signalling through proliferation-inducing pathways, such as phosphatidylinositol 3-kinase, nuclear factor kappa B, AKT and Her2 (Laughner et al, 2001; Harris, 2002; Kaluz et al, 2002; Pore et al, 2006; BelAiba et al, 2007; Kaluz et al, 2009). Highly hypoxic tumour cells are characterised by poor proliferation (Sullivan and Graham, 2007; Jubb et al, 2010; Wilson and Hay, 2011); therefore, cells exhibiting both proliferation and EMH expression could represent cells exposed to additional proliferative stimuli or alternative regulation of EHM and not necessarily diminished oxygen availability. Mildly and moderately hypoxic tumours may remain proliferative (Hoogsteen et al, 2005) and express high levels of CAIX because of the presence of aberrant cellular signalling pathways that impact HIF1 $\alpha$ stability and activity (Pastorekova et al, 2008; Kaluz et al, 2009). This suggests that the measurement of EMH within the tumour may not accurately reflect the level of tumour hypoxia.

We recently reported that CAIX expression in the tumour stroma (sCAIX) is a stronger predictor of HNSCC patient survival than CAIX expression in the tumour (Brockton et al, 2011, 2012). This may reflect the relatively normal nature of stromal cells compared with tumour cells, in which genomic instability leads to deregulation of hypoxic signalling (Ivanov et al, 1998; Wykoff et al, 2000). However, the presence of proliferative tumour cells surrounding the stroma might be a consequence of growth factor and cytokine signalling in the tumour microenvironment. These same factors can induce $\mathrm{pO}_{2}$-independent stabilisation of HIF1 $\alpha$ (Kaluz et al, 2009) and upregulate CAIX expression in the stroma. We hypothesised that proliferation-associated sCAIX expression attenuates the association between sCAIX expression and survival outcomes in OSCC. In the present study, we account for proliferation-associated, hypoxia-independent CAIX expression by incorporating Ki67 status to stratify patients with high sCAIX expression. The biological relevance of our prognostic signature is supported by its association with reduced BAX expression (Erler et al, 2004; Nelson et al, 2004). This approach identifies patients with the least favourable prognosis who would potentially benefit most from the inclusion of additional therapies to their current treatment regimens.

\section{MATERIALS AND METHODS}

Patient cohort. Institutional ethics approval was obtained from the Conjoint Health Research Ethics Board at the University of Calgary, in accordance with the Tri-Council Policy Statement on Research with Human Subjects. The retrospective study cohort has been previously described (Bose et al, 2012) and included 102 treatment-naive, surgically resected patients with OSCC diagnosed between 1998 and 2005 at the Foothills Medical Centre, Calgary, Alberta, Canada. All patients were treated by two head and neck surgeons (JCD and Dr. T Wayne Matthews). Although these patients comprise a subset of oral cancer patients diagnosed during this period, there were no significant differences in clinical and demographic characteristics between these patients and our population-based prospective clinical cohort diagnosed between 2009 and 2012, indicating that these patients are representative of the disease burden in Alberta, Canada (data not shown). Postoperative radiotherapy was included based on the presence of metastatic lymph nodes, extra-capsular spread (ECS) or positive surgical margins. A clinical outcome database and associated tissue microarray (TMA) series were constructed as described previously (Bose et al, 2012). Treatment-naive, formalin-fixed, paraffinembedded (FFPE) tumour samples were available for 87 of the 102 patients. Tissue cores suitable for the evaluation of CAIX and Ki67 were available for 60 of these 87 patients. Triplicate cores from pathologically confirmed tumour-bearing regions of each FFPE tumour block were included for each patient to account for intra-tumour heterogeneity (Camp et al, 2008). Five samples of normal oral cavity squamous epithelium, separate from the tumourcontaining regions of archived FFPE tissue blocks, were also included in the TMAs as reference samples to establish biomarker levels in normal tissue (Bose et al, 2012).

Quantitative fluorescence IHC. We used fluorescence IHC and the HistoRx AQUA platform (Camp et al, 2002) to quantify the expression of CAIX, Ki67 and BAX in each TMA core. The details of the staining conditions for CAIX and vimentin (Brockton et al, 2012), Ki67 (Klimowicz et al, 2012) and BAX (Bose et al, 2012) have been previously described. Briefly, TMA slides were stained using a 1:500 dilution of anti-vimentin (280618, rat mAb, R\&D Systems, Burlington, ON, Canada) and a $1: 1000$ dilution of antiCAIX (ab15086, rabbit pAb, Abcam, Cambridge, UK). For Ki67, a 1:5000 dilution of anti-Ki67 (MIB1, mouse mAb, Dako, Burlington, ON, Canada) was used and for Bax, a 1:5000 dilution of anti-Bax (E63, rabbit mAb, Epitomics, Burlingame, CA, USA) was used. The respective secondary antibodies, rat HRP (Santa Cruz Biotechnology, Santa Cruz, CA, USA) and mouse or rabbit Envision + (Dako) were used along with the sequential application of the fluorophores tyramide-Cy3, for detection of vimentin and Ki67 or tyramide-Cy5, for the detection of CAIX and Bax (Perkin-Elmer, Waltham; MA, USA). A 1:100 dilution of a pancytokeratin antibody (guinea pig polyclonal, Acris, San Diego, CA, USA) and an Alexa488-conjugated anti-guinea pig secondary antibody (Invitrogen, Burlington, ON, Canada) were included in 
all fluorescence IHC assays. All slides were mounted in DAPIcontaining medium (Invitrogen).

Slides were scanned using a HistoRx PM-2000 (Genoptix, Carlsbad, CA, USA) and analysed by AQUAnalysis software (version 2.2.1.7; Genoptix). The tumour compartment was defined as the PCK-positive area for each TMA core, the tumour nuclear compartment was defined as the DAPI-positive area within the tumour compartment, the Ki67 compartment area was defined as the Ki67-positive area within the tumour nuclear compartment (Klimowicz et al, 2012), and the stromal compartment was defined as the vimentin-positive and PCK-negative area (Bose et al, 2012). AQUAnalysis software was used to calculate sCAIX and tumour BAX AQUA scores representing the exposure time-adjusted and lamp-intensity normalised average pixel intensity of each biomarker within the compartment of interest. The algorithm AQUA score determination and background discrimination has been reported previously (Gustavson et al, 2009). Ki67 expression was reported as the percentage of tumour nuclear area that was positive for Ki67 (Klimowicz et al, 2012).

Statistical analysis. Patients were dichotomised by the basal Ki67 expression (percentage of tumour nuclear area positive for Ki67) to define low and high proliferation groups. Pathologists often select the least favourable region of a tumour (e.g., differentiation status) to define the whole tumour. We used this principle to apply our understanding of the biology of our candidate markers and select biologically relevant definitions. Basal Ki67 expression for each OSCC patient was defined as the lowest level measured among triplicate TMA cores; this definition of Ki67 status was most strongly associated with 5 -year disease-specific survival (DSS) in a previous evaluation of Ki67 scoring strategies (Klimowicz et al, 2012). CAIX expression in the tissue core, expressing the maximum level of sCAIX among triplicate TMA cores, was used to identify patients with top-quartile CAIX expression in the stromal compartment (sCAIX ${ }^{\text {hi }}$ ) (Brockton et al, 2012). We have previously reported that the maximum sCAIX expression associates with significantly worse 5-year DSS in patients with the highest sCAIX.

BAX expression for each patient was defined by the mean BAX expression from triplicate TMA cores. Mean BAX expression was compared in box and whisker plots and Welch's $t$-test analyses. The Fisher's exact test was used to compare clinical characteristics between patient groups defined by combined sCAIX and Ki67 expression. Kaplan-Meier curves and the logrank test were used to assess the association with 5-year DSS. Survival was measured from the date of diagnosis to the date of death or the date of last follow-up. The impact of clinically significant covariates, including pathologic $\mathrm{T}$ stage (pT-stage) and node status (N-stage), was assessed using Cox proportional hazards analyses. All statistical analyses were performed using Stata 12 (StataCorp LP, College Station, TX, USA).

\section{RESULTS}

Cohort characteristics. Our study adheres to the REMARK criteria (McShane et al, 2005). The median age of patients included in the study was 61.5 years (range: $26-83$ years). Median follow-up for the cohort was 50 months and the 5-year survival estimate was $69.6 \%$. Univariate analyses of clinico-pathological characteristics are presented in Table 1. Frozen section margins were evaluated and were clear in 58 of the 60 resections. ECS status was only reported in 11 of 28 (39\%) node-positive patients. Both pT- and N-stage were significantly associated with DSS (logrank $P<0.005)$. All patients were treated with primary surgery and 45 $(72 \%)$ received post-operative radiotherapy. Two patients received concurrent chemoradiation therapy as treatment for their primary disease but these patients were not analysed or reported separately.
Biomarker expression analysis. Representative fluorescence IHC images for each biomarker are presented in Figure 1. CAIX expression was predominantly membranous or cytoplasmic in both the tumour and stromal compartments (Figure 1A). The median intensity of sCAIX expression in OSCC tumour samples was 1379 (95\% CI 442-2315). As expected, tumour Ki67 expression was predominantly nuclear (Figure 1B). Proliferative (Ki67-positive) cells were observed both in the tumour and stromal compartments. The median basal Ki67 percent positive tumour nuclear area score in OSCC samples was $31.2 \%$ (95\% CI 17.2-65.6\%). Tumour BAX expression was predominantly cytoplasmic (Figure 1C). The median intensity of BAX expression in OSCC was 4609 (95\% CI 2972-6616).

A prognostic signature combining CAIX and Ki67 expression. As previously reported, high stromal CAIX expression $\left(\mathrm{sCAIX}^{\mathrm{hi}}\right)$ was associated with poor DSS (Brockton et al, 2011, 2012), (Figure 2A). Stratification of sCAIX ${ }^{\text {hi }}$-expressing tumours by their median basal Ki67 expression identified a group with significantly worse survival; the median survival for sCAIX ${ }^{\text {hi }}$ and Ki67 ${ }^{\text {lo }}$ sCAIX ${ }^{\text {hi }}$ groups was $\sim 38$ and $\sim 18$ months, respectively (Figure $2 \mathrm{~A}$ and $\mathrm{B}$ ). Patients identified by this signature (top-quartile CAIX expression with below-median basal Ki67 expression; $\mathrm{Ki}^{\mathrm{lo}}{ }^{\mathrm{sCAIX}}{ }^{\mathrm{hi}}$ ) exhibited significantly worse DSS compared with all other patients in the OSCC cohort (Figure 2C). Patients in the Ki67 ${ }^{\text {lo }}$ sCAIX ${ }^{\text {hi }}$ group did not differ significantly by age, gender, clinical stage, pT-stage, differentiation status, smoking history, alcohol history or treatment selection (Table 1). However, the $\mathrm{Ki}^{\mathrm{lo}}{ }^{\mathrm{sCAIX}}{ }^{\mathrm{hi}}$ group was significantly associated with positive $\mathrm{pN}$-stage $(P=0.007$; Table 1$)$. Patients in this group also exhibited a higher propensity for lymph node metastasis (89\% of patients are lymph node positive) compared with patients expressing sCAIX ${ }^{\text {hi }}$ alone (69\% of patients are lymph node positive).

The independent prognostic impact of $\mathrm{Ki}^{\mathrm{lo}} \mathrm{sCAIX}^{\mathrm{hi}}$ was assessed using Cox proportional hazards models. pT-stage (T1/T2 vs T3/T4) and pN-stage (N0 vs N1/N2) were included with $\mathrm{Ki}^{2} 7^{\mathrm{lo}} \mathrm{sCAIX}^{\mathrm{hi}}$ in the multivariate model based on their acknowledged clinical significance in OSCC and their significant association with DSS in univariate analyses (Table 1). All three variables, pT-stage (HR $1.026(1.002-1.049), P=0.032)$, pN-stage (HR $6.794(1.852-24.924), P=0.004)$ and $\mathrm{Ki}^{\mathrm{lo}}{ }_{\text {sCAIX }}{ }^{\text {hi }}$ (HR 4.209 (1.387-12.774), $P=0.011)$, were independently associated with DSS (Table 2).

Relevance of prognostic signature to hypoxia. Given that BAX expression is suppressed in hypoxia (Erler et al, 2004; Nelson et al, 2004), we analysed the association between our EMH and proliferation-based prognostic signature, and BAX expression. BAX expression was lower in the $\mathrm{Ki} 67^{\mathrm{lo}}$ sCAIX ${ }^{\mathrm{hi}}$ group compared with the rest of the cohort (Figure $3 \mathrm{~A}$ ). The difference in BAX expression was negligible when patients were stratified based on sCAIX expression alone (Figure 3B).

\section{DISCUSSION}

In this study, we address the potential confounding effect of proliferative signalling on the association between CAIX and prognosis. We demonstrate that the prognostic utility of CAIX can be improved by considering the hypoxia-independent contribution of proliferative signalling pathways to CAIX expression. Tumours characterised by low proliferation and high sCAIX expression $\left(\mathrm{Ki}^{\mathrm{lo}}{ }^{\mathrm{l}}\right.$ SCAIX $\left.{ }^{\mathrm{hi}}\right)$ were associated with significantly worse DSS in univariate and multivariate analyses. Accounting for the contribution of tumour proliferation in our CAIX-based prognostic assay (Brockton et al, 2012), by stratifying patients based on tumour ki67 status ( $\mathrm{Ki} 67^{\mathrm{lo}} \mathrm{sCAIX}^{\mathrm{hi}}$ ), identified patients with the most aggressive disease course. These patients also demonstrated the most 
Table 1. Demographic and clinico-pathological characteristics of the study cohort

\begin{tabular}{|c|c|c|c|c|c|}
\hline Characteristics & No. of cases (\%) & $\begin{array}{c}\mathrm{Ki}^{6} 7^{\mathrm{lo}} \\
\operatorname{sCAIX}^{\mathrm{hi}}(\boldsymbol{n}=9)\end{array}$ & $\begin{array}{l}\mathrm{Ki}^{6} 7^{\text {hi }} \text { or Ki67 } \\
\text { sCAIX }^{\text {lo }}(n=51)\end{array}$ & $\begin{array}{c}\text { Fisher's exact } \\
(\boldsymbol{P} \text {-value })\end{array}$ & Logrank ( $P$-value) \\
\hline \multicolumn{6}{|l|}{ Age, years } \\
\hline $\begin{array}{l}<60 \\
\geqslant 60\end{array}$ & $\begin{array}{l}28(47) \\
32(53)\end{array}$ & $\begin{array}{l}4 \\
5\end{array}$ & $\begin{array}{l}24 \\
27\end{array}$ & 0.588 & 0.534 \\
\hline \multicolumn{6}{|l|}{ Gender } \\
\hline $\begin{array}{l}\text { Male } \\
\text { Female }\end{array}$ & $\begin{array}{l}42(70) \\
18(30)\end{array}$ & $\begin{array}{l}5 \\
4\end{array}$ & $\begin{array}{l}37 \\
14\end{array}$ & 0.257 & 0.875 \\
\hline \multicolumn{6}{|c|}{ Pathologic T stage } \\
\hline $\begin{array}{l}\mathrm{T} 1+\mathrm{T} 2 \\
\mathrm{~T} 3+\mathrm{T} 4 \\
\text { Unknown }\end{array}$ & $\begin{array}{c}32(53) \\
27(45) \\
1(2)\end{array}$ & $\begin{array}{l}4 \\
5 \\
0\end{array}$ & $\begin{array}{r}28 \\
22 \\
1\end{array}$ & 0.389 & $0.004^{*}$ \\
\hline \multicolumn{6}{|c|}{ Pathologic N stage } \\
\hline $\begin{array}{l}\mathrm{N} 0 \\
\mathrm{~N} 1+\mathrm{N} 2\end{array}$ & $\begin{array}{l}32(53) \\
28(47)\end{array}$ & $\begin{array}{l}1 \\
8\end{array}$ & $\begin{array}{l}31 \\
20\end{array}$ & $0.007^{*}$ & $<0.001^{*}$ \\
\hline \multicolumn{6}{|c|}{ Differentiation status } \\
\hline $\begin{array}{l}\text { Well } \\
\text { Moderate } \\
\text { Poor } \\
\text { Unknown }\end{array}$ & $\begin{aligned} & 10(17) \\
& 35(58) \\
& 7(12) \\
& 8(13)\end{aligned}$ & $\begin{array}{l}0 \\
6 \\
2 \\
1\end{array}$ & $\begin{array}{r}10 \\
29 \\
5 \\
7\end{array}$ & 0.198 & 0.077 \\
\hline \multicolumn{6}{|l|}{ Smoking history } \\
\hline $\begin{array}{l}\text { Never } \\
\text { Ever }\end{array}$ & $\begin{array}{l}13(22) \\
47(78)\end{array}$ & $\begin{array}{l}3 \\
6\end{array}$ & $\begin{array}{l}10 \\
41\end{array}$ & 0.299 & 0.708 \\
\hline \multicolumn{6}{|l|}{ Alcohol history } \\
\hline $\begin{array}{l}\text { Never + occasional } \\
\text { Moderate + heavy } \\
\text { Unknown }\end{array}$ & $\begin{array}{r}28(47) \\
26(43) \\
6(10)\end{array}$ & $\begin{array}{l}4 \\
4 \\
1\end{array}$ & $\begin{array}{r}24 \\
22 \\
5\end{array}$ & 0.604 & 0.086 \\
\hline \multicolumn{6}{|l|}{ Treatment } \\
\hline $\begin{array}{l}\text { Surgery } \\
\text { Surgery + radiation }\end{array}$ & $\begin{array}{l}15(25) \\
45(72)\end{array}$ & $\begin{array}{l}2 \\
7\end{array}$ & $\begin{array}{l}13 \\
38\end{array}$ & 0.601 & 0.125 \\
\hline
\end{tabular}

significant decrease in BAX expression, the downregulation of which is a common feature of hypoxia, (Erler et al, 2004; Nelson et al, 2004).

We have previously shown that sCAIX is more strongly associated with patient survival than tumour CAIX (Brockton et al, 2011, 2012). CAIX expression in the stroma is not confounded by common OSCC-associated somatic mutations such as CAIX gene amplification and deletion of the von Hippel-Lindau (VHL) gene (Sparano et al, 2006). Loss of VHL, a regulator of HIF1 $\alpha$, has been shown to promote hypoxia-independent expression of CAIX (Ivanov et al, 1998; Wykoff et al, 2000). However, sCAIX expression may be affected by proliferation-associated factors that may misrepresent hypoxic status. Although we observed proliferating cells in the stromal compartment, the impact of proliferative signalling in the tumour cells is likely to be most relevant to disease course. As proliferative pathways are predominantly deregulated in the tumour rather than the stroma, we assigned proliferation status based on Ki67 in the tumour cells. Paracrine/autocine growth factor signalling within the tumour microenvironment may lead to HIF $1 \alpha$ stabilisation in the stroma and upregulate sCAIX expression independent of hypoxia. Additionally, high tumour cell density can lead to peri-cellular hypoxia due to the acute and local depletion of oxygen and nutrients, which could also cause increased sCAIX expression (Chrastina, 2003; Kaluz et al, 2009). Therefore, the combination of sCAIX and tumour Ki67 expression could identify tumours that are 'functionally hypoxic'.

It is well recognised that hypoxic tumours are generally associated with poor prognosis (Vaupel and Mayer, 2007; Jubb et al, 2010) and several groups have investigated the role of both hypoxia and proliferation in OSCC and other head and neck cancers (Ljungkvist et al, 2002; Hoogsteen et al, 2005; Kondo et al, 2011). Although a stromal CAIX and tumour Ki67 signature has not been previously explored, there is some support in the existing literature (Hoogsteen et al, 2005) for our combined hypoxia and proliferation-based prognostic signature providing a more biologically relevant definition of hypoxic tumours than sCAIX alone. Our Ki67 and CAIX-based signature identifies a population of OSCC patients with significantly worse prognosis than sCAIX alone. Furthermore, it has been reported that the poor prognosis associated with tumour hypoxia is partly attributed to hypoxiainduced tumour cell migration and invasion (Sullivan and Graham, 2007). We report that patients with poorly proliferative tumours expressing high sCAIX (Ki67 ${ }^{\text {lo }}$ sCAIX ${ }^{\text {hi }}$ ), exhibit a higher propensity for lymph node metastasis compared with patients expressing sCAIX $^{\text {hi }}$ alone. Despite this strong correlation between lymph node metastasis and $\mathrm{Ki}_{6} 7^{\mathrm{lo}}$ sCAIX ${ }^{\text {hi }}$-defined hypoxia, we demonstrate 
A
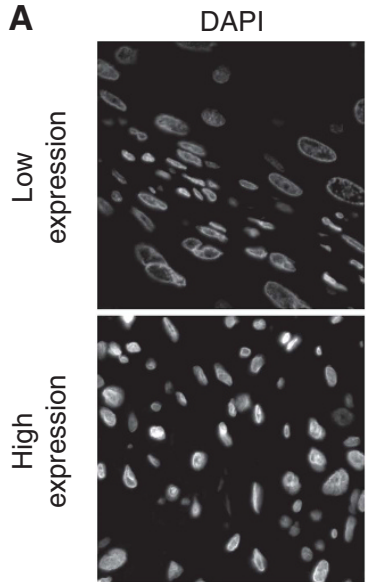

Blue
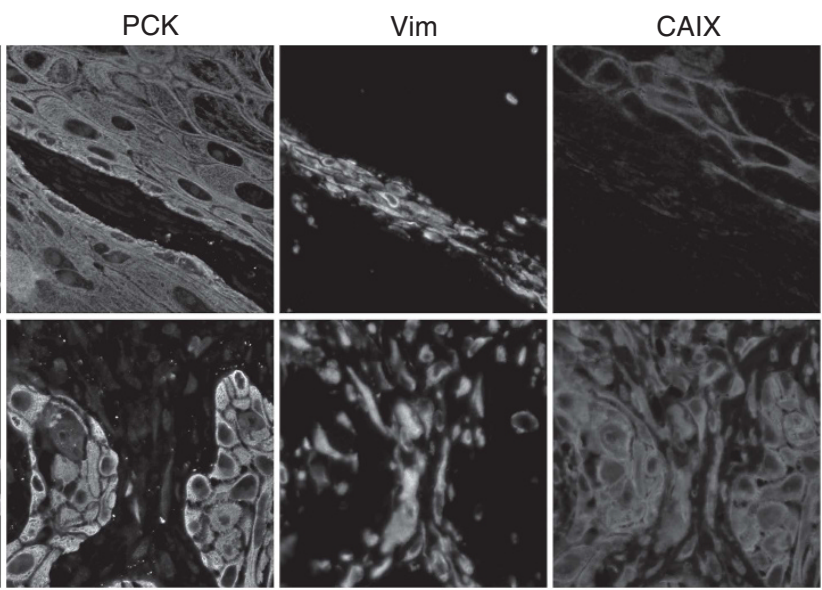

Green

B

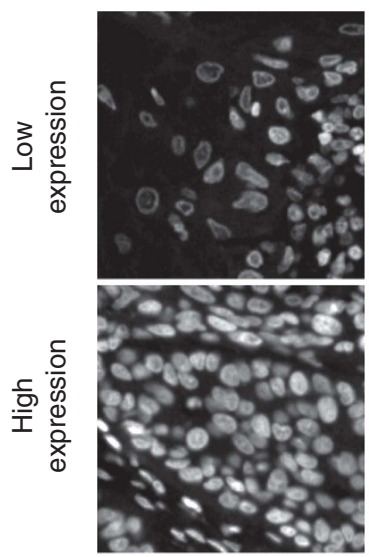

Blue

C

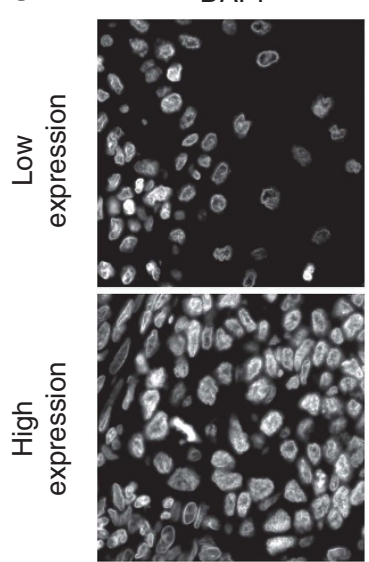

Blue

PCK

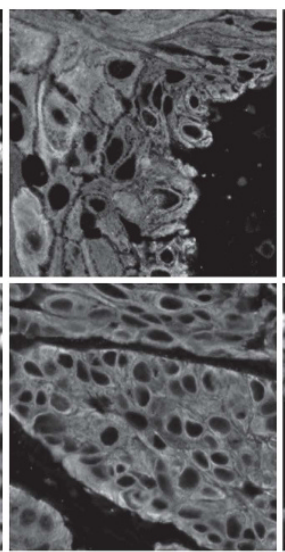

Green

PCK

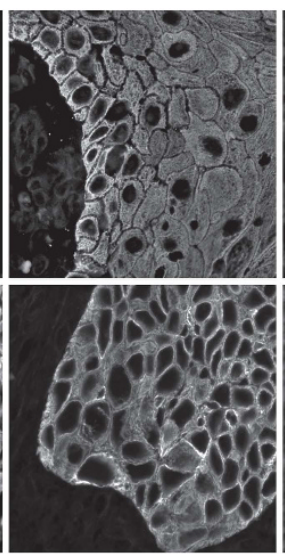

Green

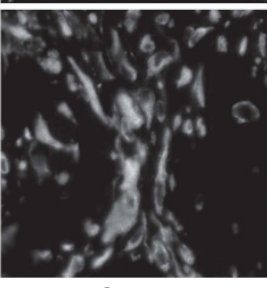

Grey

Ki67

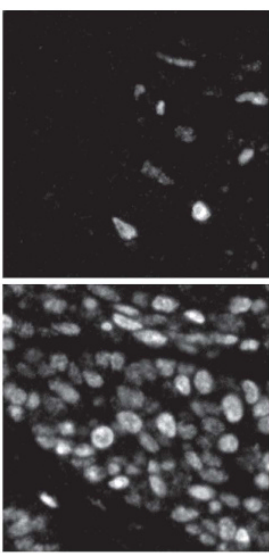

Red

BAX

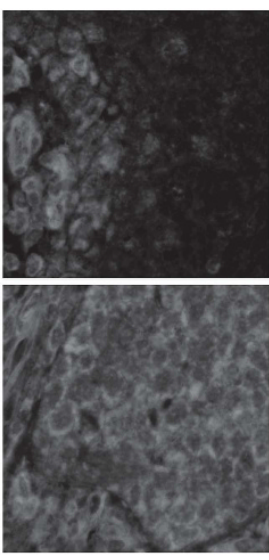

Red

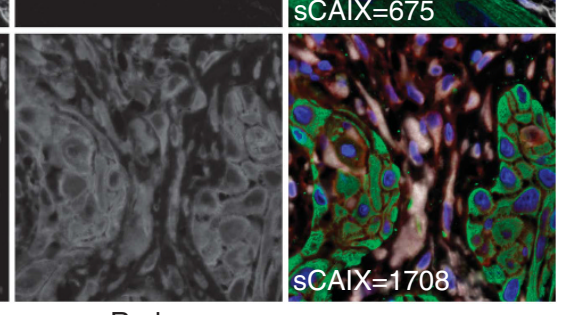

Red

Merge

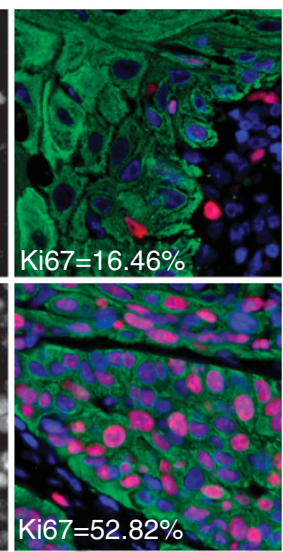

Merge

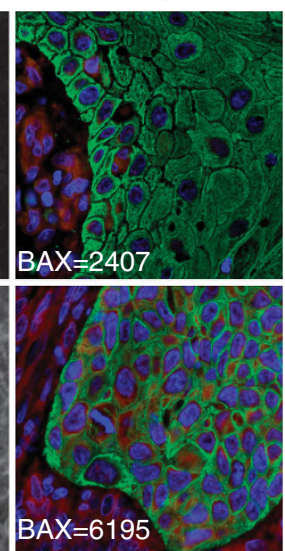

$B A X=6195$

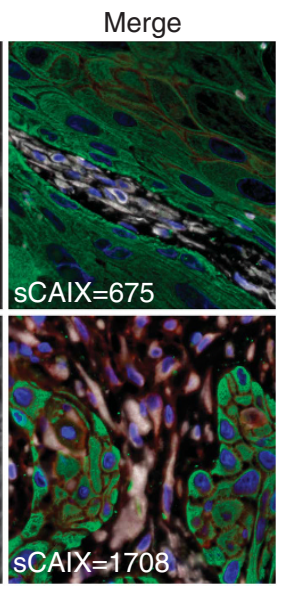

\section{1}

Figure 1. Fluorescent immunohistochemical staining using the HistoRx AQUAnalysis platform. Representative fluorescence

immunohistochemistry images for (A) sCAIX, (B) Ki67, and (C) BAX expression in OSCC samples. Upper and lower panels present examples of low and high biomarker expression, respectively. Merged images are pseudo-coloured blue for DAPI, green for pan-cytokeratin, red for the biomarker of interest, and greyscale for vimentin. AQUA scores for BAX and sCAIX and the percent positive tumour nuclear area for Ki67 are indicated in the merged images.

that $\mathrm{Ki} 67^{\mathrm{lo}}$ sCAIX ${ }^{\mathrm{hi}}$ is an independent prognostic factor in our OSCC cohort after adjusting for $\mathrm{pT}$ - and pN-stage in multivariate analysis. Lastly, hypoxic tumours have been shown to be resistant to apoptosis-inducing stimuli (Wilson and Hay, 2011). BAX expression is downregulated in hypoxia (Erler et al, 2004) and suppression of the pro-apoptotic BAX/BAK pathways is required for tumour cells to survive in hypoxic conditions and evade treatment-induced apoptosis (Nelson et al, 2004). Accordingly, we found that BAX expression was markedly lower in Ki6 $7^{\mathrm{lo}} \mathrm{SCAIX}{ }^{\mathrm{hi}}$ tumours compared with tumours defined by sCAIX ${ }^{\mathrm{hi}}$ alone. 
The observation of discrepancies between different methods for identifying hypoxic tumours has led to the development of mRNA expression-based hypoxia profiles. These profiles are reported to
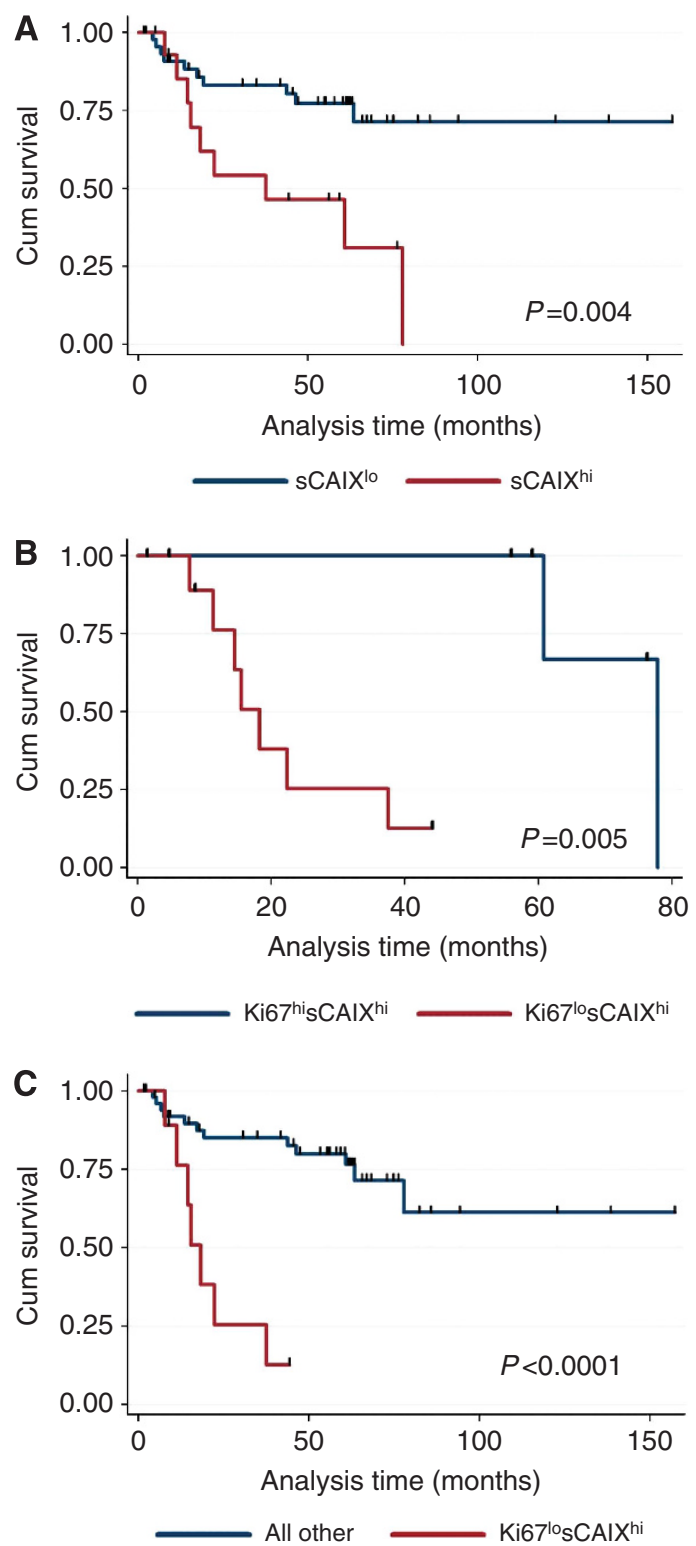

Figure 2. Univariate survival analysis of prognostic signatures in OSCC. Kaplan-Meier curves and logrank $P$-values for disease-specific survival in (A) all OSCC patients dichotomised based on SCAIX expression status, (B) OSCC patients with high sCAIX dichotomised based on Ki67 expression, and (C) all OSCC patients dichotomised based on Ki67 $7^{\text {lo }}$ SCAIX ${ }^{\text {hi }}$ status. more accurately identify hypoxic tumours by accounting for multiple hypoxia-associated factors (Toustrup et al, 2012). However, while this is promising for research applications, mRNA profile-based testing has not been broadly adopted in the clinic due to several logistical challenges. Currently, few mRNA profile-based tests are in routine clinical use for treatment selection in cancer; the most prominent of these is the Genomic Health 21-gene Recurrence Score test for predicting recurrence after anti-oestrogen therapy in early-stage breast cancer (Ross et al, 2008). However, this test may soon be replaced by a more clinically feasible fourcomponent IHC-based equivalent, IHC4 (Cuzick et al, 2011).

The two-component 'functional hypoxia' signature (Ki67 and sCAIX) proposed in this study demonstrates a significant improvement over the use of sCAIX alone for identifying patients with poor prognosis. A Ki67/sCAIX-based IHC test could be developed and routinely applied in the clinic to detect functionally hypoxic tumours and direct the use of several promising treatment strategies (Wilson and Hay, 2011; Bose et al, 2013). Also, such a test can be applied to tumours that are not treated surgically, to identify patients that will benefit from the addition of hypoxia-targeting/sensitising therapies to existing chemoradiation
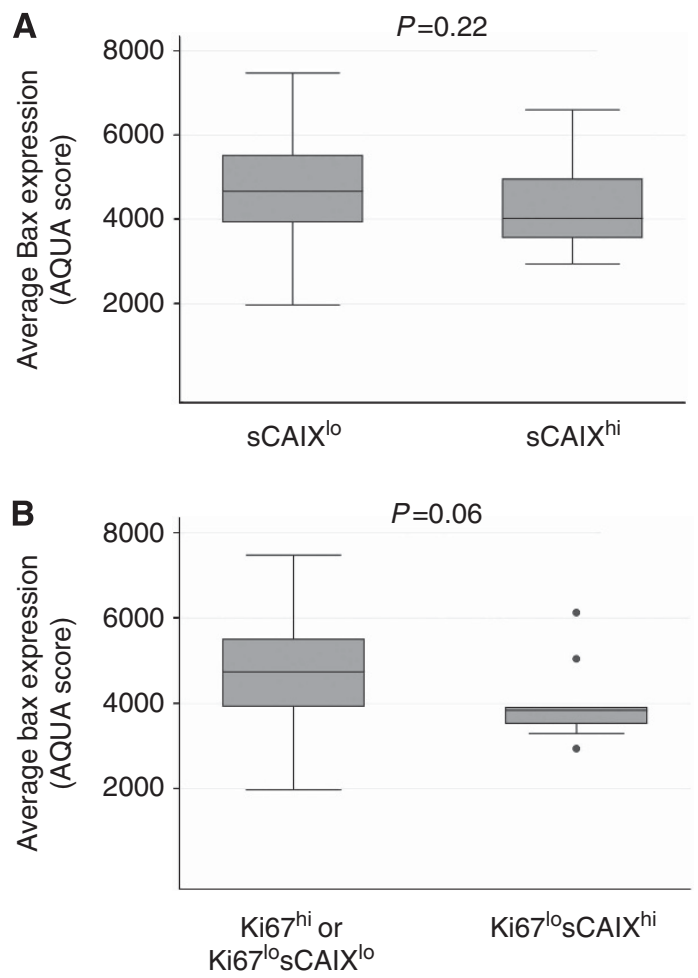

Figure 3. BAX expression analysis in patients stratified by SCAIX and Ki67 expression status. Box and whisker plots comparing BAX expression in the OSCC cohort by (A) SCAIX expression status alone or (B) Ki67 ${ }^{\text {lo }}$ sCAIX ${ }^{\text {hi }}$ status. Welch's t-test $P$-values are reported.

Table 2. Cox proportional hazards analysis of disease-specific survival

Univariate

Multivariate

\begin{tabular}{|c|c|c|c|c|}
\hline Covariates & Hazard ratio $(95 \% \mathrm{Cl})$ & $\boldsymbol{P}$-value & Hazard ratio $(95 \% \mathrm{Cl})$ & $P$-value \\
\hline $\mathrm{Ki}^{2} 7^{\mathrm{lo}}$ sCAIX ${ }^{\text {hi }}$ (no vs yes) & $7.175(2.522-20.414)$ & $<0.001$ & $4.209(1.387-12.774)$ & 0.011 \\
\hline Pathological T stage (T1/T2 vs T3/T4) & $1.010(0.990-1.031)$ & 0.315 & $1.026(1.002-1.049)$ & 0.032 \\
\hline Pathological N stage (N0 vs N1/N2) & $6.882(2.268-20.885)$ & 0.001 & $6.794(1.852-24.924)$ & 0.004 \\
\hline
\end{tabular}


regimens. Additional biomarkers could potentially be incorporated to further tailor this signature to specific tumour types (Chi et al, 2006 ) or to specific hypoxia-sensitising/targeting treatments. However, testing the inclusion of additional markers will require a larger patient cohort than the one reported in this study.

Our study is limited by its retrospective design and the lack of corroborative $\mathrm{pO}_{2}$ or pimonidazole measurements. ECS is an important prognostic factor in OSCC and its incomplete reporting at the time that these patients were treated is a further limitation of our study. Additionally, the $\mathrm{Ki}^{7} 7^{\mathrm{lo}} \mathrm{sCAIX}^{\text {hi }}$ group was relatively small, leading to the possibility of 'over-fitting' the data. This and the post-hoc definition of survival groups can both be addressed by replication in an independent OSCC cohort. However, our results strongly suggest that using combined sCAIX and tumour Ki67 expression, to account for hypoxia-independent CAIX expression, could lead to the development of a cost-effective and clinically feasible test to efficiently identify patients with unfavourable outcomes that may indicate functionally hypoxic tumours.

\section{ACKNOWLEDGEMENTS}

We thank Dr. T. Wayne Matthews for contributing patients to this study and Dr. E.M. Long for her help with editing of the manuscript and figure preparation. We also gratefully acknowledge the Ohlson Research Initiative for funding this project.

\section{REFERENCES}

BelAiba RS, Bonello S, Zahringer C, Schmidt S, Hess J, Kietzmann T, Gorlach A (2007) Hypoxia up-regulates hypoxia-inducible factor-1 alpha transcription by involving phosphatidylinositol 3-kinase and nuclear factor kappa B in pulmonary artery smooth muscle cells. Mol Biol Cell 18(12): 4691-4697.

Bose P, Brockton NT, Dort JC (2013) Head and neck cancer: from anatomy to biology. Int J Cancer 133(9): 2013-2023.

Bose P, Klimowicz AC, Kornaga E, Petrillo SK, Matthews TW, Chandarana S, Magliocco AM, Brockton NT, Dort JC (2012) Bax expression measured by AQUAnalysis is an independent prognostic marker in oral squamous cell carcinoma. BMC Cancer 12: 332.

Brockton N, Dort J, Lau H, Hao D, Brar S, Klimowicz A, Petrillo S, Diaz R, Doll C, Magliocco A (2011) High stromal carbonic anhydrase IX expression is associated with decreased survival in p16-negative head-andneck tumors. Int J Radiat Oncol Biol Phys 80: 249-257.

Brockton NT, Klimowicz AC, Bose P, Petrillo SK, Konno M, Rudmik L, Dean M, Nakoneshny SC, Matthews TW, Chandarana S, Lau HY, Magliocco AM, Dort JC (2012) High stromal carbonic anhydrase IX expression is associated with nodal metastasis and decreased survival in patients with surgically-treated oral cavity squamous cell carcinoma. Oral Oncol 48(7): 615-622.

Camp RL, Chung GG, Rimm DL (2002) Automated subcellular localization and quantification of protein expression in tissue microarrays. Nat Med 8(11): 1323-1327.

Camp RL, Neumeister V, Rimm DL (2008) A decade of tissue microarrays: progress in the discovery and validation of cancer biomarkers. J Clin Oncol 26(34): 5630-5637.

Campana JP, Meyers AD (2006) The surgical management of oral cancer. Otolaryngol Clin North Am 39(2): 331-348.

Chen L, Gajendrareddy PK, DiPietro LA (2012) Differential expression of HIF-1 alpha in skin and mucosal wounds. J Dent Res 91(9): 871-876.

Chi JT, Wang Z, Nuyten DS, Rodriguez EH, Schaner ME, Salim A, Wang Y, Kristensen GB, Helland A, Borresen-Dale AL, Giaccia A, Longaker MT, Hastie T, Yang GP, van dV, Brown PO (2006) Gene expression programs in response to hypoxia: cell type specificity and prognostic significance in human cancers. PLoS Med 3(3): e47.

Chrastina A (2003) High cell density-mediated pericellular hypoxia is a crucial factor inducing expression of the intrinsic hypoxia marker CA IX in vitro in HeLa cells. Neoplasma 50(4): 251-256.

Cuzick J, Dowsett M, Pineda S, Wale C, Salter J, Quinn E, Zabaglo L, Mallon E, Green AR, Ellis IO, Howell A, Buzdar AU, Forbes JF (2011) Prognostic value of a combined estrogen receptor, progesterone receptor, Ki-67, and human epidermal growth factor receptor 2 immunohistochemical score and comparison with the genomic health recurrence score in early breast cancer. J Clin Oncol 29(32): 4273-4278.

Erler JT, Cawthorne CJ, Williams KJ, Koritzinsky M, Wouters BG, Wilson C, Miller C, Demonacos C, Stratford IJ, Dive C (2004) Hypoxia-mediated down-regulation of bid and bax in tumors occurs via hypoxia-inducible factor 1-dependent and -independent mechanisms and contributes to drug resistance. Mol Cell Biol 24(7): 2875-2889.

Gustavson MD, Bourke-Martin B, Reilly DM, Cregger M, Williams C, Tedeschi G, Pinard R, Christiansen J (2009) Development of an unsupervised pixel-based clustering algorithm for compartmentalization of immunohistochemical expression using Automated QUantitative Analysis. Appl Immunohistochem Mol Morphol 17(4): 329-337.

Harris AL (2002) Hypoxia-a key regulatory factor in tumour growth. Nat Rev Cancer 2(1): 38-47.

Hoogsteen IJ, Marres HA, Wijffels KI, Rijken PF, Peters JP, van den Hoogen FJ, Oosterwijk E, van der Kogel AJ, Kaanders JH (2005) Colocalization of carbonic anhydrase 9 expression and cell proliferation in human head and neck squamous cell carcinoma. Clin Cancer Res 11(1): 97-106.

Hoogsteen IJ, Marres HAM, Bussink J, van der Kogel AJ, Kaanders JHAM (2007) Tumor microenvironment in head and neck squamous cell carcinomas: Predictive value and clinical relevance of hypoxic markers. A review. Head Neck 29(6): 591-604.

Ivanov SV, Kuzmin I, Wei MH, Pack S, Geil L, Johnson BE, Stanbridge EJ, Lerman MI (1998) Down-regulation of transmembrane carbonic anhydrases in renal cell carcinoma cell lines by wild-type von Hippel-Lindau transgenes. Proc Natl Acad Sci USA 95(21): 12596-12601.

Janssen HL, Haustermans KM, Balm AJ, Begg AC (2005) Hypoxia in head and neck cancer: How much, how important? Head Neck 27(7): 622-638.

Jemal A, Bray F, Center MM, Ferlay J, Ward E, Forman D (2011) Global cancer statistics. CA Cancer J Clin 61(2): 69-90.

Jubb AM, Buffa FM, Harris AL (2010) Assessment of tumour hypoxia for prediction of response to therapy and cancer prognosis. J Cell Mol Med 14(1-2): 18-29.

Kaanders JHAM, Wijffels KIEM, Marres HAM, Ljungkvist ASE, Pop LAM, van den Hoogen FJA, De Wilde PCM, Bussink J, Raleigh JA, van der Kogel AJ (2002) Pimonidazole binding and tumor vascularity predict for treatment outcome in head and neck cancer. Cancer Res 62(23): 7066-7074.

Kaluz S, Kaluzova M, Chrastina A, Olive PL, Pastorekova S, Pastorek J, Lerman MI, Stanbridge EJ (2002) Lowered oxygen tension induces expression of the hypoxia marker MN/carbonic anhydrase IX in the absence of hypoxia-inducible factor 1 alpha stabilization: A role for phosphatidylinositol 3 '-kinase. Cancer Res 62(15): 4469-4477.

Kaluz S, Kaluzova M, Liao SY, Lerman M, Stanbridge EJ (2009) Transcriptional control of the tumor- and hypoxia-marker carbonic anhydrase 9: a one transcription factor (HIF-1) show? Biochim Biophys Acta 1795(2): 162-172.

Klimowicz AC, Bose P, Nakoneshny SC, Dean M, Huang LL, Chandarana S, Magliocco AM, Matthews TW, Brockton NT, Dort JC (2012) Basal Ki67 expression measured by digital image analysis is optimal for prognostication in oral squamous cell carcinoma. Eur J Cancer 48(14): 2166-2174.

Kondo Y, Yoshikawa K, Omura Y, Shinohara A, Kazaoka Y, Sano J, Mizuno Y, Yokoi T, Yamada S (2011) Clinicopathological significance of carbonic anhydrase 9, glucose transporter-1, Ki-67 and p53 expression in oral squamous cell carcinoma. Oncol Rep 25(5): 1227-1233.

Laughner E, Taghavi P, Chiles K, Mahon PC, Semenza GL (2001) HER2 (neu) signaling increases the rate of hypoxia-inducible factor 1 alpha (HIF-1 alpha) synthesis: novel mechanism for HIF-1-mediated vascular endothelial growth factor expression. Mol Cell Biol 21(12): 3995-4004.

Ljungkvist ASE, Bussink J, Rijken P, Kaanders J, van der Kogel AJ, Denekamp J (2002) Vascular architecture, hypoxia, and proliferation in first generation xenografts of human head-and-neck squamous cell carcinomas. Int J Radiat Oncol Biol Phys 54(1): 215-228.

Mayer A, Hockel M, Vaupel P (2005) Carbonic anhydrase IX expression and tumor oxygenation status do not correlate at the microregional level in locally-advanced cancers of the uterine cervix. Clin Cancer Res 11(20): 7220-7225.

McDonald PC, Winum JY, Supuran CT, Dedhar S (2012) Recent developments in targeting carbonic anhydrase IX for cancer therapeutics. Oncotarget 3(1): 84-97. 
McShane LM, Altman DG, Sauerbrei W, Taube SE, Gion M, Clark GM (2005) Reporting recommendations for tumor marker prognostic studies. J Clin Oncol 23(36): 9067-9072.

Nelson DA, Tan TT, Rabson AB, Anderson D, Degenhardt K, White E (2004) Hypoxia and defective apoptosis drive genomic instability and tumorigenesis. Genes Dev 18(17): 2095-2107.

Pastorekova S, Ratcliffe PJ, Pastorek J (2008) Molecular mechanisms of carbonic anhydrase IX-mediated $\mathrm{pH}$ regulation under hypoxia. BJU Int 101: 8-15.

Pore N, Jiang ZB, Shu HK, Bernhard E, Kao GD, Maity A (2006) Akt1 activation can augment hypoxia-inducible factor-1 alpha expression by increasing protein translation through a mammalian target of rapamycin-independent pathway. Mol Cancer Res 4(7): 471-479.

Ross JS, Hatzis C, Symmans WF, Pusztai L, Hortobagyi GN (2008) Commercialized multigene predictors of clinical outcome for breast cancer. Oncologist 13(5): 477-493.

Sobhanifar S, Aquino-Parsons C, Stanbridge EJ, Olive P (2005) Reduced Expression of Hypoxia-inducible factor- $1 \alpha$ in perinecrotic regions of solid tumors. Cancer Res 65(16): 7259-7266.

Sorensen BS, Hao J, Overgaard J, Vorum H, Honore B, Alsner J, Horsman MR (2005) Influence of oxygen concentration and $\mathrm{pH}$ on expression of hypoxia induced genes. Radiother Oncol 76(2): 187-193.

Sowter HM, Raval RR, Moore JW, Ratcliffe PJ, Harris AL (2003) Predominant role of hypoxia-inducible transcription factor (Hif)-1alpha versus
Hif-2alpha in regulation of the transcriptional response to hypoxia. Cancer Res 63(19): 6130-6134.

Sparano A, Quesnelle KM, Kumar MS, Wang Y, Sylvester AJ, Feldman M, Sewell DA, Weinstein GS, Brose MS (2006) Genome-wide profiling of oral squamous cell carcinoma by array-based comparative genomic hybridization. Laryngoscope 116(5): 735-741.

Sullivan R, Graham CH (2007) Hypoxia-driven selection of the metastatic phenotype. Cancer Metastasis Rev 26(2): 319-331.

Toustrup K, Sorensen BS, Alsner J, Overgaard J (2012) Hypoxia gene expression signatures as prognostic and predictive markers in head and neck radiotherapy. Semin Radiat Oncol 22(2): 119-127.

Vaupel P, Mayer A (2007) Hypoxia in cancer: significance and impact on clinical outcome. Cancer Metastasis Rev 26(2): 225-239.

Wilson WR, Hay MP (2011) Targeting hypoxia in cancer therapy. Nat Rev Cancer 11(6): 393-410.

Wykoff CC, Beasley NJP, Watson PH, Turner KJ, Pastorek J, Sibtain A, Wilson GD, Turley H, Talks KL, Maxwell PH, Pugh CW, Ratcliffe PJ, Harris AL (2000) Hypoxia-inducible expression of tumor-associated carbonic anhydrases. Cancer Res 60(24): 7075-7083.

(c) (1)(2) This work is licensed under the Creative Commons Attribution-NonCommercial-Share Alike 3.0 Unported License. To view a copy of this license, visit http://creativecommons. org/licenses/by-nc-sa/3.0/ 